\title{
Experimental rheology of model colloidal dispersions Jorrit Mellema
}

Experimental rheological aspects of colloidal dispersions are now being investigated with improved skills and with better defined model systems. New methods allow more detailed investigations. The relationship between the rheological macroproperties, microproperties and processes is the focus of the most recent investigations. Better insight has been gained concerning this relationship.

\section{Addresses \\ Rheology Group, JM Burgers Centrum, Faculty of Applied Physics, University of Twente, PO Box 217, 7500 AE Enschede, The Netherlands; e-mail: J.Mellema@tn.utwente.nl}

Current Opinion in Colloid \& Interface Science 1997, 2:411-419

Electronic identifier 1359-0294-002-00411

(c) Current Chemistry Ltd ISSN 1359-0294

Abbreviations

PHSA poly-12-hydroxy stearic acid

PMMA poly methyl methacrylate

\section{Introduction}

Over the past decade, the rheological aspects of colloidal dispersions have become better mapped out, due to the development of model dispersions, improved rheological techniques, adequate modeling and the application of nonrheological techniques to investigate microstructures and processes. The general program to study the relationship between the rheological macroproperties, the microproperties and processes, faces numerous parameters which can influence the rheological behavior. At the particle level, the size and volume fraction $\phi$ are the first to be considered, and a lot of work has been done on nearly monodisperse spherical particles (say, with radius a).

Polydispersity is a complicated factor of colloidal dispersions and investigations concerning this subject are few and far between. During recent years, the study of a few bidisperse systems have been reported $[1,2,3 \cdot, 4]$. The inclusion of a shape distribution would complicate the studies very much. Unless stated otherwise, this review is restricted to nearly monodisperse spheres. The interparticle forces between particles are very important and the present review will be focussed along this line. In addition, there are thermodynamic force fields due to the position and orientation distribution of the particles and hydrodynamic forces are always present. The interplay of force fields and applied flow fields leads to microstructures and processes which can influence the rheological behavior drastically. The search for classification of microproperties and processes according to flow field strength is ongoing. Due to the fact that the type of flow field influences the microproperties and processes it is unfortunate that the type of flow field is usually shear, because a well defined extensional flow for dispersions is still not an option.

\section{Figure 1}

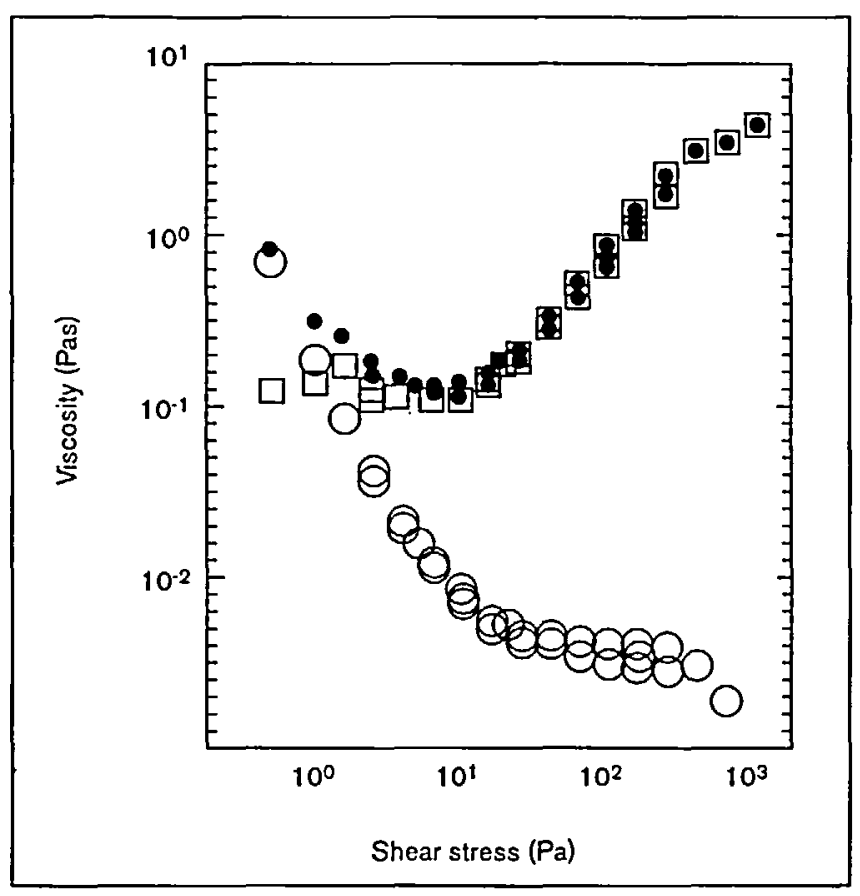

Components of the shear viscosity as resolved from the optical-stress law suspension of nearly hard spheres at $\phi=0.65$. Symbols: $=\eta$ total; $\left\ulcorner=\eta^{\text {hydro; }} O=\eta^{\text {thermo. }}\right.$. Multiple points represent the up and down branches of the flow curve demonstrating reversibility. Reproduced with permission from [ $3^{\circ}$ ].

This review is divided into two quite different regimes: near and far from equilibrium. It focuses on the literature of the past three years.

\section{Near equilibrium}

For small deformations, a colloidal dispersion displays linear viscoelastic behavior. This behavior can be represented by the complex shear modulus $G^{*}$ as a function of the applied oscillatory angular frequency $\omega$, or alternatively by the complex viscosity $\eta^{*}$ defined by $G^{*}=i \omega \eta^{*}$, with $\mathrm{i}^{2}=-1$. In a sufficiently weak steady shear flow (the rate of shear $\dot{\gamma} \rightarrow 0)$ the following relationship holds $\eta(\dot{\gamma} \rightarrow 0)=\eta^{\prime}$ $(\omega \rightarrow 0)$ between the steady state viscosity and the real part of the complex viscosity. The basic question is still how these quantities vary with the parameters mentioned in the introduction with the search generally seeking simple scaling rules. A classical question is what the relationship is between the viscosity $\eta^{\prime}$ for $\omega \rightarrow 0$ or $\omega \rightarrow \infty$ and an equilibrium quantity such as a diffusion coefficient. 


\section{Hard spheres}

Two competing systems are models for hard spheres (i.e. no interparticle force except for an infinite repulsion at contact). The first contains silica spheres either sterically stabilized by octadecyl alcohol chains grafted to the surface in cyclohexane, or simply index marched in ethylene glycol/glycerol. The second contains poly methyl methacrylate (PMMA) spheres stabilized with a grafted layer of poly-12-hydroxy stearic acid (PHSA) dispersed in decaline/tetraline.

The linear viscoelastic behavior is only mapped out for silica particles $[5,6]$. In several respects the observed behavior is equivalent to older work $[7,8]$ though the high frequency limit is not yet settled. Recently, a flattening off of the storage modulus $G^{\prime}$ at high frequency was observed [5] for index matched silica spheres, in contrast to earlier observations for sterically stabilized spheres $[7,8]$ where the storage modulus increased as the square root of frequency. This difference is most important for the interpretation because a finite asymptote is expected when hydrodynamic interactions are important. The question still remains as to how hard the particles are (in [5], index matching was not perfect and in [6] it was concluded that there is also electrostatic repulsion). Also, the use of time-temperature superposition [5] complicates the interpretation. In [5], the correlation $D_{s}(\phi) / D_{0}=\eta_{0} / \eta_{\infty}$ was found to hold up to a volume fraction of $\phi=0.55$. Here, $D_{0}=k T / 6 \pi \eta_{0} a$ and $\mathrm{kT}$ is the thermal energy, $\eta_{0}$ is the viscosity of the continuous phase, and $D_{s}$ is the short time self-diffusivity. This relationship is in agreement with other work (see e.g. [9]) and is considered in detail in [10].

Most interesting is a cautious claim in [11 $\left.{ }^{\circ}\right]$ that data from a dispersion of PMNA/PHSA spheres may represent the hard sphere curve for low shear viscosities as a function of volume fractions up to 0.5 . These data differ from those of silica spheres and would seriously affect the interpretations in $[9,10,12]$. The hardness of the spheres is mainly based on a comparison of the osmotic pressure with the Carnahan-Stirling equation. Considerable effort has been devoted to the determination of the volume fraction from the freezing transition, yielding an interaction volume fraction in contrast to the hydrodynamic volume fraction determined in [5-8] and also in a related paper [13]. Comparison of the PMMA and silica viscosities shows that there is agreement for $\eta(\dot{\gamma} \rightarrow 0)$ up to $\phi=0.4$ and for $\eta(\dot{\gamma} \rightarrow \infty)$ up to $\phi=0.6$ and only disagreement for $\eta(\dot{\gamma} \rightarrow 0)$ between $\phi=0.4$ and 0.5 (PMMA/PHSA higher). Thus simple explanations of systematic errors in the $\phi$ determination or polydispersity do not suffice. It is puzzling that the PMMA/PHSA particles are also known to behave as more or less soft particles [1,14]. After rescaling of the volume fraction to obtain an effective hard sphere diameter (in a certain way the softness of the surface is taken into account), the viscosity curves in [14] are closer to (but higher than) the PMMA/PHSA curves of [11 $\left.{ }^{\circ}\right]$ than to those of the silica particles. Again, the difference is obvious for $\eta(\dot{\gamma} \rightarrow 0)$ between $\phi=0.4$ and 0.5 . It will take some time to sort out the differences so the question of what the 'real' low shear viscosity for hard spheres is yet to be answered.

For the PMMA hard sphere dispersions also the long time diffusion coefficient $D$ describing structural relaxation has been measured with dynamic light scattering [15]. The correlation $D_{0} / D(\phi)=\eta(\dot{\gamma} \rightarrow 0) / \eta_{0}$ was found to hold up to $\phi=0.5$. For the long time self-diffusion coefficient $D_{L}$ the correlation $D_{0} / D_{L}(\phi)=c \eta(\dot{\gamma} \rightarrow 0) / 6 \eta_{0}$ was deduced with $c$ varying from 6 to 4 with increasing $\phi$ up to 0.5 [15]. The first correlation is not understood. The latter is interpreted in terms of change from stick to slip boundary condition of the effective medium at the surface of a single particle.

\section{Repulsive spheres \\ Electrostatic}

Electrostatically repulsive spheres order at sufficiently low electrolyte concentration and sufficiently high volume fraction, otherwise remaining as a single disordered phase or a two phase system.

The linear viscoelastic behavior of colloidal crystals is still a subject of study. Usually, latices are the model $[4,16,17 \cdot, 18]$, but charged, stabilized silica spheres $[19,20]$ are interesting as well. In the ordered state $G^{\prime}$ is constant over many decades of frequency: A low frequency transition in $G^{\prime \prime}$, a hollow curve at the high frequency side approaching $\omega \eta_{0}$, shows that the constancy of $G^{\prime}$ does not necessarily indicate solid behavior $\left[17^{\bullet}, 19\right]$ although the meaning is still unresolved. Modeling with the proper expression of $G^{\prime}$ unifies all experimental data in one master curve, and shows that in almost all cases $G^{\prime}$ is independent of the surface charge density [16].

When $G^{\prime}$ is constant, it is often related to a nonlinear quantity: a dynamic yield stress $\tau$ which is observed at low rates of shear. The ratio $\tau / G^{\prime}$ is approximately constant and has a magnitude of a few hundredths. This ratio is related to the critical shear at which nonlinearity occurs, and also to the Lindemann factor for melting. The magnitude and the interpretation of the ratio depends on the definition of $\tau$ and detailed studies [18] show its intricacies.

At sufficiently high electrolyre and/or low volume concentration the ordering breaks down. This takes place in a narrow regime of these parameters as can be seen in a phase diagram shown in [17*]. Then a Newtonian plateau appears at low rates of shear and at low frequencies.

When the volume fraction is corrected for the influence of the electrical double layer, the behavior of an electrostatically stabilized dispersion of charged spheres at high electrolyte is similar to that of hard spheres [2]. It is suggested in [2] that the normalized long time self-diffusion coefficient $D_{L}$ equals the inverse normalized viscosity at low shear rates for spheres smaller than $100 \mathrm{~nm}$. 


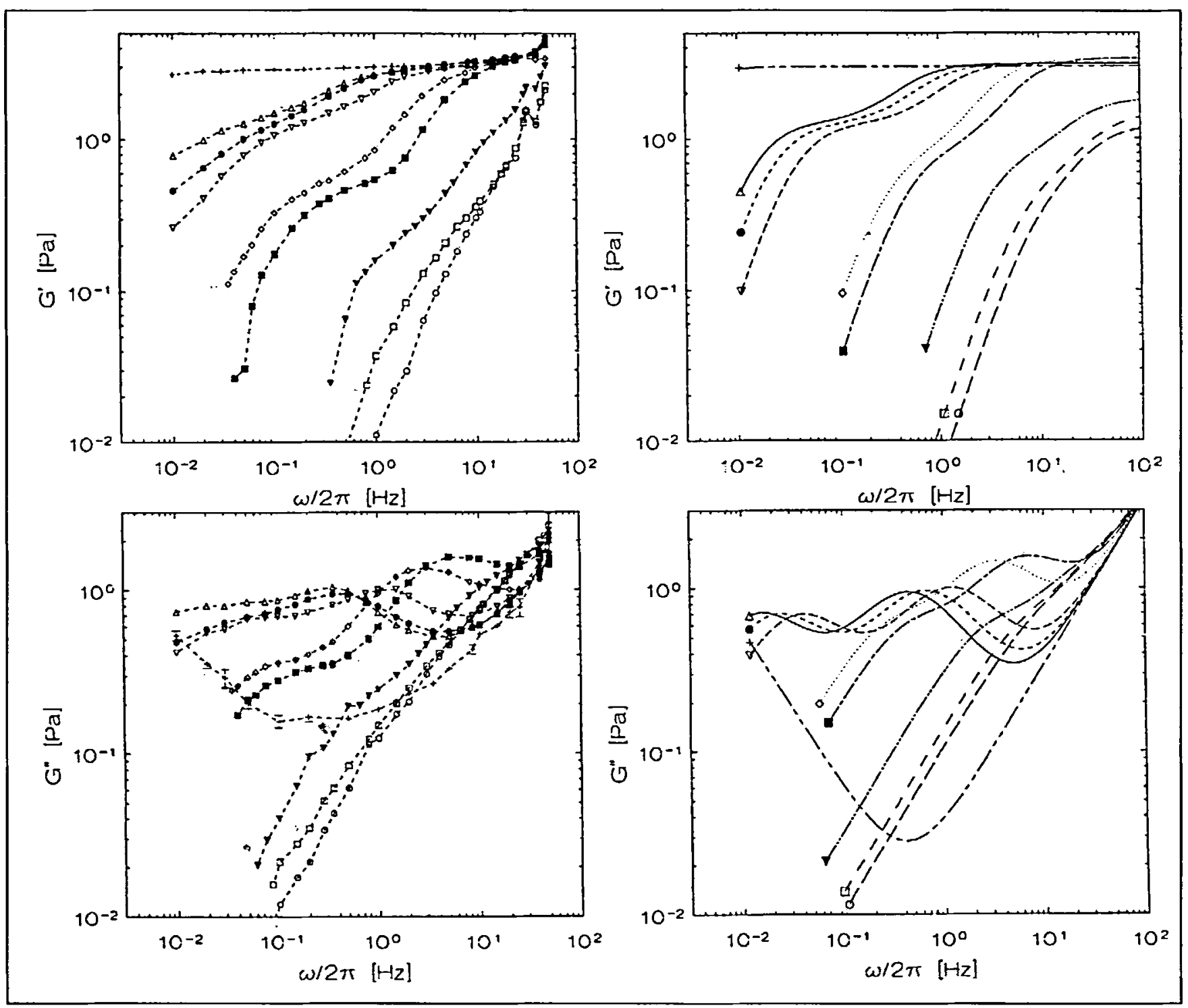

The storage modulus (upper two figures) and the loss modulus (lower two figures) for a colloidal crystal of highly deionized spheres in water as a function of the frequency for a volume fraction $\phi=0.19$. The two figures on the left-hand side show the experimental results and the figures on the right-hand side show the modeling of the assumed underlying structural changes. The applied shear rates are as follows: $(+) 0 s^{-1} ;(\triangle)$ $0.0125 \mathrm{~s}^{-1}$; (O) $0.025 \mathrm{~s}^{-1}$; (V) $0.05 \mathrm{~s}^{-1}$; (0) $0.5 \mathrm{~s}^{-1}$; (D) $1.25 \mathrm{~s}^{-1} ;(V) 5 \mathrm{~s}^{-1} ;(0) 10 \mathrm{~s}^{-1}$; (O) $25 \mathrm{~s}^{-1}$. Reproduced with permission from [170].

A careful study of this relationship carried out in [20] does not support the equality, though a relationship between $\eta(\dot{\gamma} \rightarrow 0)$ and $D_{L}$ is explicable in terms of the contribution of the continuous phase and the other particles to the friction coefficient of one particle moving in a dispersion, as was also done later in [15] for hard spheres.

\section{Hairy}

There is an increasing interest for (soft) hairy, sterically stabilized particles. Hairy particles can be made by adsorption or grafting of longer molecules to the surfaces of particles. When they are short and densely packed and dispersed in the proper solvent they behave similar to hard particles. When the hairs are longer and/or they are less densely packed, effects of deformability and interactions of 'hairs' can be expected. The layer thickness $L$, compared to the radius a of the core and the core $\phi$ are relevant parameters. Questions to be addressed are, for example, for what values of $\mathrm{a} / \mathrm{L}$ and $\phi$ does the behavior resemble that for hard spheres, and how interpenetration of hairs influences the rheological behavior.

Recently studied hairy particles are polystyrene latices stabilized by adsorbed polyvinyl alcohol $(\mathrm{a} / \mathrm{L}=2)$ [21] and an ABA blockcopolymer ( $\mathrm{A}$ : polyethylene oxide, $\mathrm{B}$ : polypropylene oxide, $a / \bar{L}=20-50$ and 3) [22]; PMMA sterically stabilized by grafted PHSA chains $(\mathrm{a} / \mathrm{L}=7-50)$ $[1,23]$; silica spheres sterically stabilized with grafted poly 
dimethyl siloxane $(\mathrm{a} / \mathrm{L}=1.4)$ ( $\mathrm{MH}$ Buijnink, PA Nommensen, MHG Duits, D van den Ende, J Mellema, unpublished data).

Experimentally, the state of art is simply to map out the rheological behavior as models are scarce. Recently, theory has addressed a few aspects of the influence of interaction ([24]; SL Elliott, WB Russel, XIIth International Congress on Rheology, Quebec, Canada, 18-23 August 1996) which might allow more pointed questions to be addressed. The most interesting phenomena occur when the $\phi$ is so high that the brushes touch and begin to interpenetrate each other. As they hinder each other sterically, interpenetration may depend on time scale and flow history (MH Buijnink, PA Nommensen, MHG Duits, D van den Ende, J Mellema, unpublished data). At high concentration, interpenetration cannot be avoided and a temporary network arises generating plateaus in $G^{\prime}$ at relatively low frequencies.

\section{Attractive spheres}

Without special precautions, colloidal spheres generally attract each other by Van der Waals forces. Silica spheres covered with octadecyl chains behave as hard spheres in benzene at sufficiently high temperature, but are adhesive at lower temperature [25]. Also, in decalin or tetradecane octadecyl silica particles are attractive $\left[26^{\circ}\right]$. By adsorption of surfactants to the surface of latices, the depth of the Van der Waals potential can be reduced. With these spheres, a reversible aggregating dispersion can be made [27-29], but attraction can also be induced between hard spheres by adding polymers (depletion flocculation) to the continuous phase of a hard sphere dispersion [30]. Such spheres then form aggregates that can lead to a space filling network.

The sticky silica spheres at low temperature [25] clearly show a divergence in the longest relaxation time in the linear viscoelastic response. The temperature at which this occurs was identified as a transition from a fluid to a gel or solid state. The adhesive hard sphere diagram, represented in terms of the Baxter interaction parameter (a dimensionless temperature) and the volume fraction has a percolation transition in the vicinity of this sol-gel transition. The corresponding zero frequency viscosity could not be measured with the same apparatus.

Some progress has been made on the understanding of the power law dependence on the volume fraction of the zero rate of shear viscosity and the shear modulus at high frequencies for a space filling network of aggregating spheres [27-29]. The classical paper of Kantor and Webman [31], predicting the network elasticity for singly connected centrally interacting spheres was based on bending elasticity between spheres, which is absent for centrally interacting spheres. With multiply connected chains, it was possible [27] to introduce bending elasticity in chains of centrally interacting spheres. Further, a network theory with kinetics for stiffness changes, was applied to provide the temporality of the network. The crowning piece of a complete model was the assumption that aggregates and chains can be characterized with geometrical parameters of a self-similar structure [29]. Theory and experiments concerning $\eta(\dot{\gamma} \rightarrow O)$ and $G^{\prime}(\omega \rightarrow \infty)$ corroborate each other, though the shortcoming in the model is that the frequency dependence of $G^{*}$ was poor. An obvious extension of the model with a distribution of chain thicknesses repairs this and obtains fair agreement with experiments [30].

At volume fractions beyond 0.3 , the assumption of self similar structure begins to fail as clusters then contain only a few particles. Nevertheless, for higher $\phi$ similar power law behavior persists for $G^{\prime}\left[26^{\circ}\right]$. The kinetics of growth of $G^{\prime}$ after a certain shear history and the limit of nonlinearity was also monitored [26.]. The most interesting result is that $G^{\prime}$ is correlated with the $\phi$ at the gel point: the threshold $\phi$ at which an infinite cluster is formed. The observed proportionality agrees with the predictions for percolation networks. This interesting experimental paper makes clear that the structural background of elasticity over the whole $\phi$ range is worth further study.

Noteworthy is the claim that critical enhancement of the shear viscosity has been observed for depletion flocculating colloidal dispersions [32॰]. Measurements have been performed in the one-phase region approaching the spinodal along a dilution line, where the concentration ratio of colloid/polymer, and the interaction between the particles, are constant. The divergence of the low shear viscosity is as strong as the divergence of the correlation length.

\section{Rods}

Colloidal rods have been synthesized recently [33] from boehmite needles by precipitating silica on the surface and subsequently grafting with stearyl alcohol. The needles are dispersed in cyclohexane. They have no significant charge and are rigid. The intrinsic viscosity of this isotropic dispersion of rods is in agreement with the theoretical value for a dilute hard rod dispersion, but the volume fraction dependence of the viscosity does not. This is probably due to weak short range interactions that cause separation of layers on the time scale of months. This work, however, gives hope that we are closer to obtaining a model dispersion of hard rigid rods.

\section{Far from equilibrium}

In practice, the most important flow behavior is at finite rate of shear. The common study of the viscosity as a function of rate of shear is not simple because it is often influenced by its' flow history, and it also may take time to reach a steady state. The fact that the viscosity often differs at each rate of shear implies that the microprocesses in the fluid depend on the rate of shear. The microprocesses in flow are determined by properties of the constituents and the structures formed. The central question is how the viscosity depends on the 
rate of shear and how this can be explained in terms of microproperties and processes. The same holds for other rheological quantities. The trend is to complement direct rheological measurements with other experimental methods that probe the microstructure. Thus one is less dependent on ad hoc modeling, apart from the inspiring information from numerical simulations. As microscopic processes and structures are the basic topics, this last part of the review will introduce the more recent literature, via a few phenomena and concepts, on microprocesses and structures. Obviously, in this short article, we cannot do justice to the rich detail found in many of the papers.

\section{Shear thinning}

The most commonly observed phenomenon is that the viscosity decreases with increasing rate of shear. The long term goal to unravel what happens at the micro·level, can be approached along several lines. For dispersions, a new line is to split up stress into the part due to hydrodynamics ('viscous' part) and that due to thermodynamic forces ('elastic' part), and to relate their relative contribution to microstructure. The application of superimposed oscillations onto a steady shear is also new for dispersions.

\section{(Nearly) hard spheres}

Nearly hard sphere silica particles demonstrate that a stress-optical relationship also exists for dispersion [34]. This offers the possibility of distinguishing between the hydrodynamic and the thermodynamic parts of the stress tensor, as the latter is proportional to the $45^{\circ}$ flow dichroism $n^{\prime \prime} 45^{\circ}$. The particles, silica particles coated with (3-trimethoxy silylpropyl)-methacrylate suspended in near index matched solvent may exhibit weak surface electrostatic interactions at high volume fractions $[3,34]$. With this technique, it could be demonstrated that the hydrodynamic part of the viscosity stays almost constant in the shear thinning regime while the thermodynamic part falls down dramatically from being dominant at the lowest shear rate to almost negligible at the highest shear rate before shear thickening begins.

Similar behavior was found for other nearly hard spheres in suspension: PMMA sterically stabilized with PHSA [35']. The elastic and viscous parts were measured by another newly developed rechnique [36] utilizing cessation of steady shear. Immediately after cessation of flow the hydrodynamic stress disappears and the elastic stress is retained. The only problem is how fast one can measure the retained stress requiring extrapolation to zero time of cessation. The results are equivalent to those deduced by the stress optical law technique. The latter was complemented [34] by neutron scattering experiments showing that increasing shear rate in the shear thinning regime prior to the shear thickening produced no long range ordering. The cautious conclusion might be that the constancy of the hydrodynamic part of the viscosity correlates with a disordered particle distribution.
Figure 3

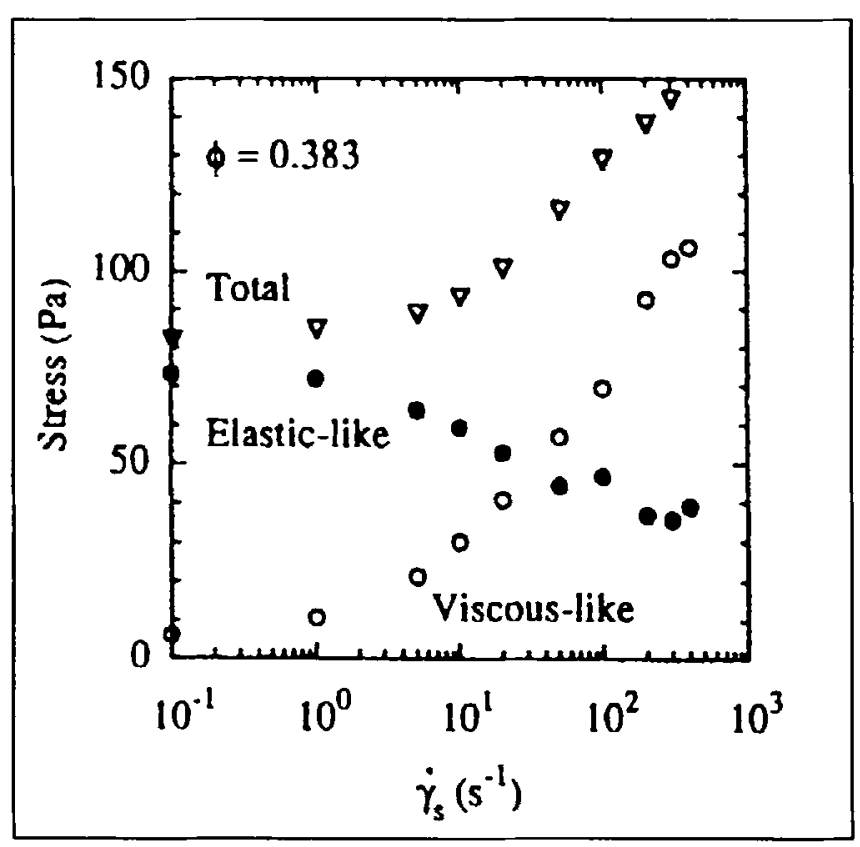

The total, elastic-like and viscous-like stress components as a function of shear rate determined with the stress jump method. The particles were charged spheres highly deionized. Reproduced with permission from [36].

\section{Repulsive spheres \\ Electrostatic}

Many studies have been conducted for electrostatically stabilized particles $[17 \bullet, 18,19,36-41]$ mostly highly deionized to give order at rest.

At relatively low salt concentration and not too high $\phi$ charged colloids may still disorder, but with a high viscosity at low shear rates. The understanding of the shear thinning behavior in these systems is an important issue for engineering purposes. Shear thinning, described in terms of an underlying microscopic activation process model, is the theme in [40]. The shear rate and $\phi$ dependence can be fairly well described as can the influence of diameter and surface potential.

Basically the same issue was addressed as part of a broader investigation on ordering latices [17 $]$, that also considered disorder caused by flow, via the formalism of transient network theory. The latter could only be tested indirectly.

Colloidal crystals under shear show a rich spectrum of phenomena shown in [18] through a nonequilibrium 'phase' diagram. The structural order depends on the Peclet number and $\phi$ of latices at a certain electrolyte concentration. Rhcology with small angle neutron scattering provided this information, as one highlight in a series of recent studies [18,37-39]. The richness of the experimental work is hard to summarize and can only be properly appreciated by careful study. 
The flow of electrostatically stabilized aqueous silica dispersions [41] can also be divided in certain regimes related to microprocesses, though different from latices. What is stressed in this study is the occurrence of wall or internal slip layers for Peclet numbers smaller than one along with the role of osmotic pressure [41].

The usefulness of a polarizing microscope was demonstrated in another study of charged silica spheres [19]. Between crossed polarizers, multiple Bragg scattering identifies crystalline pieces making shear melting nicely visible. In the cuvette configuration, coexistence of polycrystalline regions and sliding layers with less long range order was observed. The picture emerges that at sufficiently high shear rates, ordered domains move in a matrix of disordered fluid. Such as model describes the shear thinning of colloidal crystals in shear flow $\left[1^{\circ}\right]$ replacing the subtle ordering changes at low shear rates observed in [18] with shear rates in thin layers between ordered domains. At higher shear rates, the amount of disordered fluid grows at the cost of the ordered domains. This model works quite well. A new method for dispersions has been developed to superimpose oscillations orthogonally onto a steady shear flow. Thus one probes the structural changes by the determination of $\mathrm{G}^{*}$ at a certain shear rate [42]. This method was used to probe ordering domains flowing in disordered material and corroborates the microstructural picture proposed in [17 $\left.{ }^{\circ}\right]$.

Application of the stress jump method to ordered crystals under shear flow [36] reveals that both the viscous part and the elastic part of the viscosity decrease with shear rate. The elastic part dominates at low shear and the viscous part at high shear rates. As the viscous part changes drastically (a factor of 200 over more than three decades of rate of shear) the structure apparently changes a lot. It is doubtful that such an effect can be explained by subtle changes in crystal structure during flow, as observed in [18].

\section{Hairy}

The hairy particles have already been mentioned previously in this review [1,21-23]. They exhibit flow curves similar to those for hard spheres, at low $\phi$. At concentrations where the hairs can interact with each other, an apparent yield stress-like behavior appears at low rates. It is possible to determine the viscosity at high rates of shear. This high shear rate viscosity was calculated in [24] from a model for particles bearing hairy surface layers. There is some agreement between this modeling and the experiments. It is clear, however, that more experiments are necessary for the testing of the models.

\section{Attractive particles}

Aggregating dispersions have strong shear thinning behavior as well. Polystyrene latex particles with surfactant adsorbed onto their surfaces aggregate due to Van der Waals attraction [27]. Sterically stabilized silica particles in dispersion aggregate due to depletion flocculation when polymers are added to the continuous phase $[30,43]$. The flow curves can be explained quite well $[29,30,43]$ with the following model. The at-rest space filling network is assumed to be composed of aggregates with a self-similar structure; the aggregates are conceived as impermeable spheres. The size of a sphere in flow is determined by a breakup criterion that balances the strength of an aggregate against the average hydrodynamic force exerted at the outside of the aggregate. Interaggregate forces are taken into account by a transient network theory. The success of this modeling led to its application in the description of compaction during flow [43] and the sedimentation (and hydrodynamic diffusion) of aggregates in a Couette flow [44]. Structural changes during flow have been probed by superimposed oscillation onto the shear flow [30].

\section{Bidisperse system}

The most important paper on bidisperse colloidal dispersions is [1], supported by others $[2,3 \cdot, 4]$. Replacing a minor amount of large PMINA particles by smaller PMINA particles, keeping the total $\phi$ constant has a large influence on the viscosity, contrary to the other way around. This is explained by the deformability of the PMIMA particles yielding a procedure to calculate the limiting viscosities for low and high shear for monodisperse systems.

\section{Shear thickening}

Shear thickening is defined as the increase of the viscosity with the increase of rate of shear. In recent literature, it has been reported for electrostatically stabilized spheres $\left[1,6,18,37-39,45^{\circ}\right]$, sterically stabilized spheres $\left[3^{\circ}, 46\right]$ and sticky spheres [47].

The scaling of the onset of thickening is not yet clarified but systematic variation of the volume fraction, size of the particles and the interaction between the particles, is forming a picture. The additional help of nonrheological methods provides information on possible pertaining microstructures.

\section{(Nearly) hard spheres}

For the nearly index matched silica particles used in [6] for the study of linear viscoelastic behavior, the nonlinear behavior demonstrated continuous shear thickening. To probe the structure before and during shear thickening, nonlinear stress relaxation and viscosity growth and decay functions were studied. Though some considered these systems to be hard sphere systems in [6] a short ranged electrostatic interaction had to be assumed to explain certain experimental data. An interpretation balancing Brownian diffusion and hydrodynamic forces suggested an onset of thickening at a rate of shear of approximately the inverse of the weight-averaged relaxation time in the linear regime, thus it depended on $a^{-3}$. The thinning behavior below thickening is attributed to a fairly insensitive anisotropy of the particle distribution, and not 
to flow-induced order. The relaxation of the viscosity decay function in the thickening regime might be in keeping with cluster formation.

Cluster formation as the origin of shear thickening is the focus of other work [3*] exploiting the stress-optical relationship [34]. The particles that were used were silica particles coated with (3-trimethoxysilylpropyl)-methacrylate suspended in near index matching solvent.

The investigations in [3॰] mainly focus on rather abrupt shear thickening. It is shown that in the shear thickening regime the stress is predominantly hydrodynamic and that the turbidity is strongly increasing. The interpretation of these observations is that hydroclusters are formed. Neutron scattering provides no evidence for crystalline, oriented polycrystalline or string-like phases before shear thickening. The onset of thickening is related to the ratio of the Brownian force and the lubrication force based on the effective viscosity of the whole dispersion. Thus, the critical stress for onset is proportional to $\mathrm{a}^{-3}$.

Sterically stabilized particles studied in [46] corroborate the conclusion of the foregoing study for abrupt shear thickening, that the onset of thickening correlates with a critical shear stress. The experiments could not clarify whether there was $\mathrm{a}^{-2}$ or $\mathrm{a}^{-3}$ dependence. It emphasizes that the shear thickening of these sterically stabilized particles is intimately related to the nature of the particle surface.

\section{Repulsive spheres}

The electrostatically stabilized.latex particles in [18,37-39] at relatively low electrolyte concentrations (ordering at higher $\phi>0.38$ ), displayed thickening only at higher $(\phi>0.4-0.5)$ volume concentration and at shear rates where ordering is degraded. The strength of the shear thickening approaching $\phi=0.6$ was such that irreversible flocculation occurred. The interpretation of the onset of thickening is derived from a balance of stresses. Here one balances a typical elastic stress and a typical lubrication stress. As the first is chosen to be proportional to the shear modulus at rest, the radius dependence of the critical shear rate is not a simple power dependence of radius a. For a similar system, the influence of gap size and shear history was investigated [38]. Usually, clustering is believed to occur at the onset of shear thickening. Abrupt thickening is argued, on the basis of experiments with different gap sizes, to arise from a jamming together of clusters to a spanning cluster. It would also explain erratic stresses observed for this kind of shear thickening.

Anorher light has been shed [45.] on the microstructure in the shear thickening regime by small angle neutron scattering on an electrostatically stabilized acrylic latex dispersion. Upon flow cessation, a metacrystalline state developed from the disordered shear-thickened state. It is suggested that the apparently disordered shear thickened state contains a high degree of latent order.
More information on the system and the experiment is needed to evaluate its meaning.

\section{Attractive spheres}

The thickening behavior of aggregating particles [47] is less often studied. Spherical silica, coated with stearyl alcohol dispersed in benzene becomes attractive at low temperature. Experiments conducted around the gel point [47] nicely show a variety of rather abrupt changes. Weak shear thickening is observed for low rates of shear close to the gelation line and below. Light scattering indicates the formation of large, elongated structures, parallel to the flow direction. The average size become larger when the attraction between particles becomes stronger. At higher shear rates the structures are broken down.

\section{Conclusions}

Methodologically three new developments have been reported. A stress-optical law and a stress jump method allow determination of the thermodynamic part of the stress. Oscillation superimposed onto a steady shear flow permit probing of structural changes during flow.

The question concerning a standard for hard spheres is raised again by work on PMIM/PHSA spheres, which at least at higher concentration, behave as deformable weakly repulsive spheres, and also by work on index matched silica spheres. They challenge the older work on sterically stabilized silica spheres.

For repulsive spheres that order at low electrolyte concentration understanding of $G^{\prime}$ seems more or less complete.

Systematic work on hairy particles is now emerging and the first modeling is reported.

The elasticity of aggregating spheres shows interesting differences in behavior but a unified picture is not available. The interpretation of the flow of aggregates with self-similar structures has been quite successful.

A model for shear thinning based on a microscopic activation process model seems to work for electrostatically repulsive spheres. The flow of ordered colloids is investigated in great detail and a sequence of microscopic changes with increasing rate of shear is proposed. In the shear thinning regime, prior to shear thickening, long range order of string or layers is not necessarily present.

Shear thickening is distinguished as strong and weak thickening with strong meaning that the flow is more or less blocked. Convincing evidence has now been given for the occurrence of clusters formed by hydrodynamic forces in the case of strong thickening. The scaling of the onset of thickening is still subject of research.

On the whole, it is clear that our understanding of the rheology of colloids has improved. 


\section{References and recommended reading}

Papers of particular interest, published within the annual period of review, have been highlighted as:

- of special interest

-. of outstanding interest

1. D'Haene P, Mewis J: Rheological characterization of bimodal colloidal dispersions. Rheol Acta 1994, 33:165-174.

2. Richtering $\mathrm{W}$, Müller $\mathrm{H}$ : Comperison between viscosity and diffusion in monodisperse and bimodal colloidal suspensions. Langmuir 1995, 11:3699-3704.

3. Bender JW, Wagner NJ: Reversible shear thickening in - monodisperse and bidisperse colloidal dispersions. J Rheol 1996, 40:899-916.

Experimental method to separate 'viscous' and 'elastic' parts of the stress tensor. Nearly hard sphere systems were used and no long range order before shear thickening was found. Hydroclusters formed during thickening.

4. Berend K, Richtering W: Rheology of concentrated mono disperse and bidisperse polymer latices. Colloid Sur 1995 99:101-119.

5. Shikata T, Pearson DS: Viscoelastic behavior of concentrated spherical suspensions. J Rheo/ 1994, 38:601-616.

6. Watanabe $H$, Yao M-L, Yamagishi A, Osaki $K$, Shikata T, Niwa $H$, Morishima Y: Nonlinear rheological behavior of a concentrated spherical silica suspension. Rheol Acta 1996, 35:433-445.

7. Mellema J, de Kruif CG, Blom C, Viij A: Hard sphere colloidal dispersions: mechanical relaxation pertaining to thermodynamic forces. Rheol Acta 1987, 26:40-44.

8. Van der Werff JC, de Kruif CG, Blom C, Mellema J: Linear viscoelastic behavior of dense hard sphere dispersion. Phys Rev $A$ 1989, 39:795-807.

9. Brady JF: Model hard sphere dispersions: statistical mechanical theory, simulations and experiments. Curr Opin Colloid Interface Sci 1996, 1:472-480.

10. Verberg R, de Schepper IM: Viscosity of colloidal suspensions. Phys Rev E 1997, 55:3143-3159.

11. Phan SE, Russel WB, Cheng $Z$, Zhu J, Chain PM, Dunsmuir JH,

- Ottewill RH: Phase transition, equation of state, end limiting shear viscosity of hard sphere dispersions. Phys Rev E 1996, 54:6633-6645.

Challenge of sterically stabilized silica spheres as a model of hard spheres by PMMA spheres.

12. Lionberger RA, Russel WB: A Schmoluwchowski theory with simple approximations for hydrodynamic interactions in concentrated dispersions. J Rheol 1997, 41:399-425.

13. Van der Werff JC, de Kruif CG: Hard sphere colloidal dispersions: the scaling of rheological properties with particles size rate and volume fraction. J Rheol 1989, 33:4521-4547.

14. Raynaud L, Ernst B, Verge C, Mewis J: Rheology of aqueous latices with adsorbed stabilized layers. J Colloid Interface $S c i$ 1996, 181:11-19.

15. Segre PN, Meeker SP, Pusey PN, Poon WCK: Viscosity and structural relaxation in suspensions of hard sphere colloids. Phys Rev Lett 1995, 75:958-961.

16. Van der Vorst B, van den Ende D, Mellema J: Linear viscoelastic properties of ordered latices. J Rheol 1995, 33:1 183-1200.

17. Van der Vorst B: Rheology of ordering polystyrene latex - dispersions [PhD Thesis]. University of Twente: The Netherlands; 1996.

Demonstration of the probing of structural changes of ordered latices by superimposed oscillation onto steady shear.
18. Chow MK, Zukoski CF: Nonequilibrium behavior of dense suspensions of unitorm particles: volume fraction and size dependence of theology on microstructure. $J$ Rheol 1995, 39:33-59.

19. Imhof A, van Blaaderen A, Dhont JKG: Shear melting of colloidal crystals of charged spheres studied with rheology and polarizing microscopy. Langmuir 1994, 10:3477-3484.

20. Imhof A, van Blaaderen A, Maret G, Mellema J, Dhont JKG; A comparison between the long-time self-diffusion and low shear viscosity of concentrated dispersions of charged colloidal silica spheres. $J$ Chem Phys 1994, 100:2170-2181.

21. Neuhausler S, Richtering W: Rheology and diffusion in concentrated sterically stabilized polymer dispersions. Colloid Surf $A$ 1995, 97:39-51.

22. Faers MA, Luckham PF: Rheology of polyethylene oxidepolypropylene oxide block copolymer stabilized latices and emulsions. Colloid Surf A 1994, 86:317-327.

23. D'Haene P: Rheology of polymerically stabilized suspensions [PhD Thesis]. Katholieke Universiteit Leuven; Belgium; 1992.

24. Potanin AA, Russel WB: Hydrodynamic interaction of particles with grafted polymer brushes and applications to rheology of colloidal dispersions. Phys Rev 1995, 52:730-737.

25. Woutersen ATJM, Mellema J, Blom C, de Kruif CG: Linear viscoelasticity in dispersions of adhesive hard spheres. $J$ Chem Phys 1994, 101:542-553.

26. Reub CJ, Zukoski CF: Viscoelastic properties of colloidal gels. - J Rheol 1997, 41:197-218.

Scaling of elasticity for gels up to high volume fractions.

27. De Rooij R: Rheology of weakly aggregating polystyrene latex dispersions [PhD Thesis]. University of Twente; The Netherlands; 1994.

28. De Rooij R, van den Ende D, Duits MHG, Mellema J: Elasticity of weakly aggregating polystyrene latex dispersions. Phys Rev $E$ 1994, 49:3038-3049.

29. Potanin AA, de Rooil $R$, van den Ende $D$, Mellema d: Microrheological modeling of weakly aggregated dispersions. $J$ Chem Phys 1995, 102:5845-5853.

30. Wolthers W: Rheology of depletion flocculated colloidal dispersions [PhD Thesis]. University of Twente; The Netherlands; 1997.

31. Kantor $Y$, Webman I: Elastic properties of random percolating systems. Phys Rev Lett 1994, 52:1891-1894.

32. Bodnar I, Dhont JKG: Strong critical enhancement of the - shear viscosity of colloidal systems. Phys Rev Lett 1996, 77:5304-5307.

The question of whether there is critical enhancement of the shear viscosity must be kept in mind.

33. Wierenga AM, Philipse AP: Low-shear viscosities of dilute dispersions of colloidal rodlike silica particles in cyclohexane. J Colloid Interface Sci 1996, 180:360-370.

34. Bender JW, Wagner NJ: Optical measurements of the contributions of colloidal forces to the rheology of concentrated suspensions. J Colloid Interface Sci 1995, 172:171-184.

35. Kaffashi B, O'Brien VT, Mackey ME, Underwood SM: Elastic-like - and viscous-like components of the shear viscosity for nearly hard sphere, Brownian suspensions. J Colloid Interface Sci 1997, 187:22-28.

Stress jump method to separate 'viscous' and 'elastic' parts of the stress tensor for nearly hard spheres.

36. Mackey ME, Kaffashi B: Stress jumps of charged colloidal suspensions, measurements of the elastic-like and viscouslike stress components. J Colloid Interface Sci 1995, 174:117-123. 
37. Chen LB, Chow MK, Ackerson BJ, Zukoski CF: Rheological and microstructural transitions in colloidal crystals. Langmuir 1994, 10:2817-2829.

38. Chow LB, Zukoski CF: Gap size and shear history dependencies in shear thickening of a suspension ordered at rest $J$ Rheol 1995, 39:15-32.

39. Fagan ME, Zukoski CF: The rheology of charge stabilized silica suspensions. J Rheol 1997, 41:373-397.

40. Ogawa A, Yamada H, Matsuda S, Okajima K, Doi M: Viscosity equation for concentrated suspensions of charged colloidal particles. J Rheol 1997, 41:769-786.

41. Persello J, Magnin A, Chang J, Piau JM, Cabane B: Flow of colloidal aqueous silica dispersions. J Rheol 1994, 38:1845-1870.

42. Zeegers J, van den Ende D, Blom C, Altena EG. Beukema GJ, Mellema J: A sensitive dynamic viscometer for measuring the complex shear modulus in a steady shear flow using the method of orthogonal superposition. Rheol Acta 1995, 34:606-614.

43. Wolthers W, Duits MHG, van den Ende D, Mellema J: Shear history dependence of the viscosity of aggregated colloidal dispersions. J Rheol 1996, 40:799-811.

44. Wolthers W, van den Ende D, Duits MHG, Mellema J: The viscosity and sedimentation of aggregating colloidal dispersions in a Couette flow. $J$ Rheol 1996, 40:55-67.

45. Butera RJ, Wolf MS, Bender J, Wagner NJ: Formation of highly - ordered colloidal microstructure upon flow cessation from high shear rates. Phys Rev Lett 1996, 77:2117-2120.

The question of whether latent order exists in the shear thickening regime should be kept in mind.

46. Frith WJ, D'Haene P, Buscall R, Mewis J: Shear thickening in model dispersions of sterically stabilized particles. $J$ Rheol 1996, 40:531-548.

47. Verduin $H$, de Gans BJ, Dhont JKG: Shear induced structural changes in a gel-forming suspension studied by light scattering and rheology. Langmuir 1996, 12:2947-2955. 